\title{
Evaluation of performance of the Omni mode for detecting video capsule endoscopy images: A multicenter randomized controlled trial
}

Authors

Institutions
Naoki Hosoe ${ }^{1}$, Kenji Watanabe ${ }^{2}$, Takako Miyazaki², Masaaki Shimatani ${ }^{3}$, Takahiro Wakamatsu ${ }^{3}$, Kazuichi Okazaki $^{3}$, Motohiro Esaki ${ }^{4}$, Takayuki Matsumoto ${ }^{5}$, Takayuki Abe ${ }^{6}$, Takanori Kanai ${ }^{7}, K^{2}$ azuo Ohtsuka ${ }^{8}$, Mamoru Watanabe ${ }^{8}$, Keiichi Ikeda ${ }^{9}$, Hisao Tajiri ${ }^{9}$, Naoki Ohmiya ${ }^{10}$, Masanao Nakamura ${ }^{11}$, Hidemi Goto ${ }^{11}$, Tomoyuki Tsujikawa ${ }^{12}$, Haruhiko Ogata'

Institutions are listed at the end of article. submitted

28. December 2015 accepted after revision 13. June 2016

\section{Bibliography}

DOI http://dx.doi.org/

10.1055/s-0042-111389

Published online: 8.8.2016

Endoscopy International Open

2016; 04: E878-E882

(c) Georg Thieme Verlag KG

Stuttgart · New York

E-ISSN 2196-9736

\section{Corresponding author}

\section{Haruhiko Ogata, MD, PhD}

Center for Diagnostic and Therapeutic Endoscopy, School of Medicine, Keio University 35 Shinanomachi Shinjuku

Tokyo 160-8582

Japan

Phone: +81-3-3353-1211

Fax: +81-3-3357-2778

hogata@keio.jp
Background and study aims: Olympus recently developed a new algorithm called Omni mode that discards redundant video capsule endoscopy (VCE) images. The current study aimed to demonstrate the non-inferiority of the Omni mode in terms of true positives (TPs) and the superiority of the Omni mode with regard to reading time against a control (ordinary ES-10 system).

Patients and methods: This multicenter prospective study included 40 patients with various small bowel diseases. VCE images were evaluated by 7 readers and 3 judging committee members. Two randomly allocated readers assessed the VCE images obtained using the 2 modalities for each patient. The order of the modalities was switched between the 2 readers and the interval between readings by the same reader was 2 weeks. The judging committee predefined clinically relevant

\section{Introduction}

$\nabla$

Video capsule endoscopy (VCE) plays a significant role in diagnosis and management of small bowel diseases; its usefulness has been previously reported in numerous studies [1-3]. The noninvasive nature of VCE has resulted in its application in examination of other organs, including the esophagus [4], stomach [5], and colon [6]. For VCE, patients are required to swallow a video capsule. The VCE image readers must then assess more than 80,000 images, an uncomfortable and time-consuming process. VCE systems have been developed by several companies, notably Given imaging and Olympus. These companies have introduced several features to the CE software in an effort to reduce the time required to analyze VCE images and minimize the possibility of missing lesions [7-9]. Recently, Olympus developed a new algorithm called Omni mode that aims to reduce the number of redundant images and display all areas captured by VCE. lesions as major lesions and irrelevant lesions as minor lesions. The number of TPs for major and minor lesions and the reading times were compared between the modalities. The predefined non-inferiority margin for the TP ratio of the Omni mode compared with the control was 0.9.

Results: The estimated TP ratios and 95\% confidence intervals for total, major, and minor lesions were $0.87(0.80-0.95), 0.93(0.83-1.04)$, and 0.83 (0.74-0.94), respectively. Although non-inferiority was not demonstrated, the rate of detection of major lesions was not significantly different between the modalities. The reading time was significantly lower when using the Omni mode than when using the control.

Conclusions: The Omni mode may be only appropriate for the assessment of major lesions.

The aim of the current study was to demonstrate the non-inferiority of Omni mode against a control (EC-10 system, Olympus, Tokyo) in terms of the number of true positives (TPs) in all major and minor lesions, as well as its superiority for required reading time. To the best of our knowledge, this is the first multicenter prospective study to evaluate the performance of the newly developed Omni mode.

\section{Patients and methods}

$\nabla$

\section{Study design}

This multicenter prospective study was approved by the ethics committee of each participating institution and was registered with a registry approved by the International Committee of Medical Journal Editors (UMIN ID000010976). Written informed consent was obtained from all patients enrolled in the study. Patient enrollment and VCE were performed at 6 hospitals in Japan: The Jikei University Third Hospital, Nagoya Uni- 


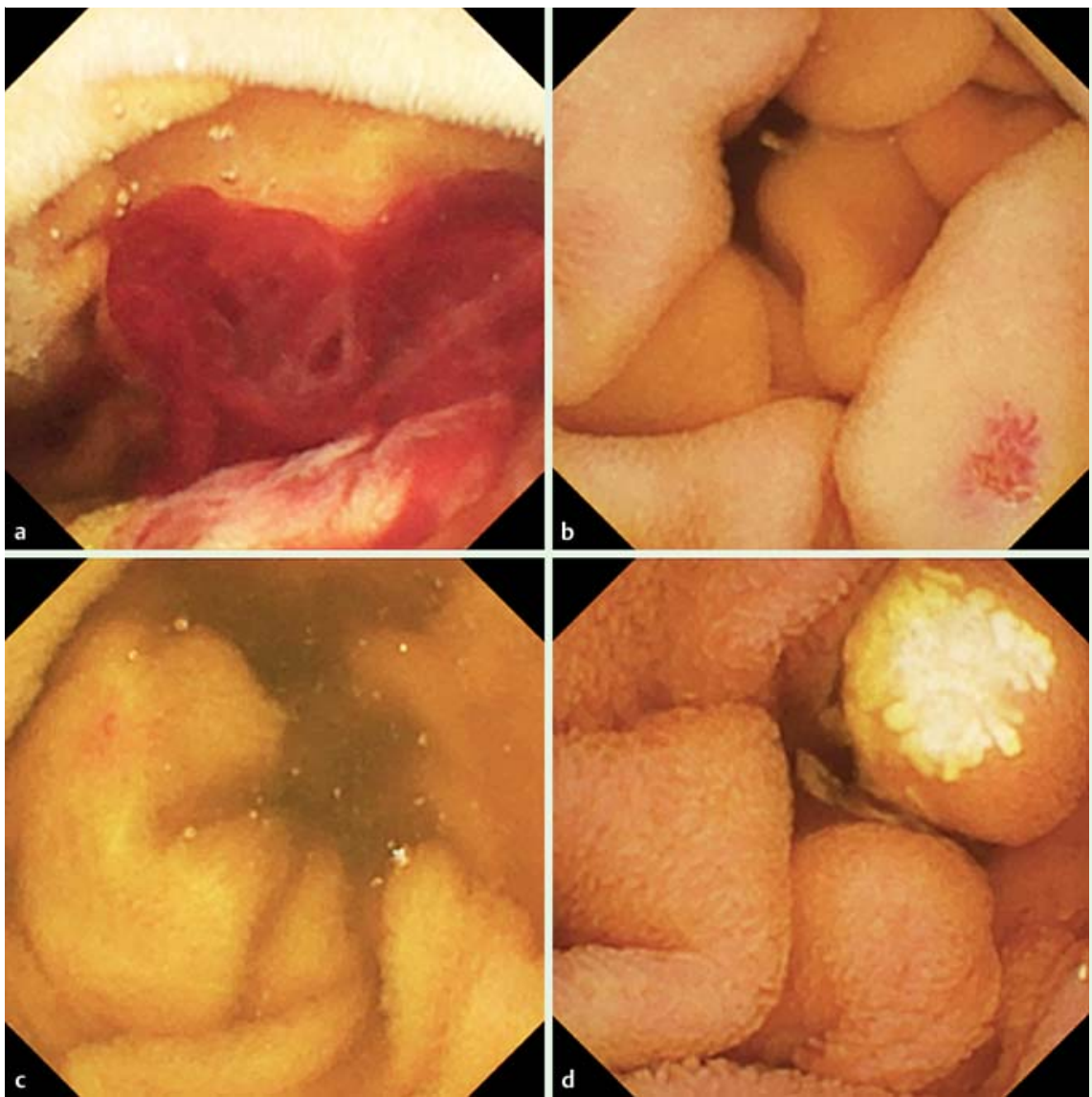

Fig. 1 Video capsule endoscopy images of predefined lesions. a Bleeding (predefined as a major lesion). b Angioectasia (predefined as a major lesion). c Angioectasia (predefined as a minor lesion). d Lymphangiectasia (predefined as a minor lesion).

versity Hospital, Kansai Medical University Hirakata Hospital, Osaka City University Hospital, Shiga Hospital, and Kyushu University Hospital. Sixty patients with suspected small bowel diseases were prospectively enrolled in this study. All patients underwent VCE and the resulting images were collected. The study utilized 7 expert VCE image readers (K.O., K.I., M.N., T.M., T.W., T.T., and M.E.) who had previously performed more than 200 VCE examinations each and 3 judging committee members (N.H., N.O., and M.S.) who had previously performed more than 200 CE examinations each. The judging committee members excluded 20 VCE videos from the 60 collected, due to the absence of lesions in the small intestine (the sample size calculation is described below). Videos that were included in the study were evaluated by the judging committee members and each lesion was defined and documented as "major" or "minor" by consensus. Clinically relevant lesions that required further examination were predefined as major lesions. These included lesions with a high potential for gastrointestinal bleeding or with uncertain bleeding potential that required further examination and intervention such as biopsy or confirmation of bleeding risk (such as ulcers, large angioectasia, massive bleeding from uncertain origin, ulcer scars, ulcer scars with stenosis, large submucosal tumors, large hemangiomas, or erosion with clots). Clinically irrelevant lesions (such as red spots, lymphangiectasia, erosion, tattooing, and small lymphangiomas) were predefined as minor lesions ( Fig. 1). Two randomly allocated readers assessed the VCE images obtained using both Omni mode and the control in each patient. All readers were blinded to the medical histories of the patients. A total of 4 readings were performed per patient. The order of the modalities was switched between the 2 readers and the interval between readings by the same reader was 2 weeks. In addition, the number of readings performed by each reader was balanced within each hospital and for each modality. Readers were allocated to assess the images according to a random schedule generated by a biostatistician (T.A.) and were blinded to the allocation. The number of correctly detected TPs and reading times were then compared between the 2 modalities.

\section{VCE procedure and reading}

The VCE procedures were performed using an EC-10 capsule and Endocapsule 10 system (Olympus, Tokyo, Japan). VCE was performed after an 8-hour fasting period. Bowel preparation and prokinetics were not defined in the protocol. The recorded digital information was downloaded from the recorder to a computer and the images analyzed using proprietary software. The first duodenal and cecal images in each video were marked by the judging committee in advance. Each reader assessed the video using dual view at a rate of 10 frames per second. As the Omni mode has the ability to select and discard redundant images, the passing of the images becomes faster than that of the control. The low frame rate was considered appropriate for reading by Omni mode, based on the results of the preliminary evaluation and in order to avoid missing lesions.

The VCE reading time was defined as the interval between the appearance of the first duodenal image and the first cecal image and was recorded. The VCE reading results were assessed and the number of TPs was tallied by the judging committee. A TP was defined as the detection of a lesion by the reader that had also been identified by the judging committee. 


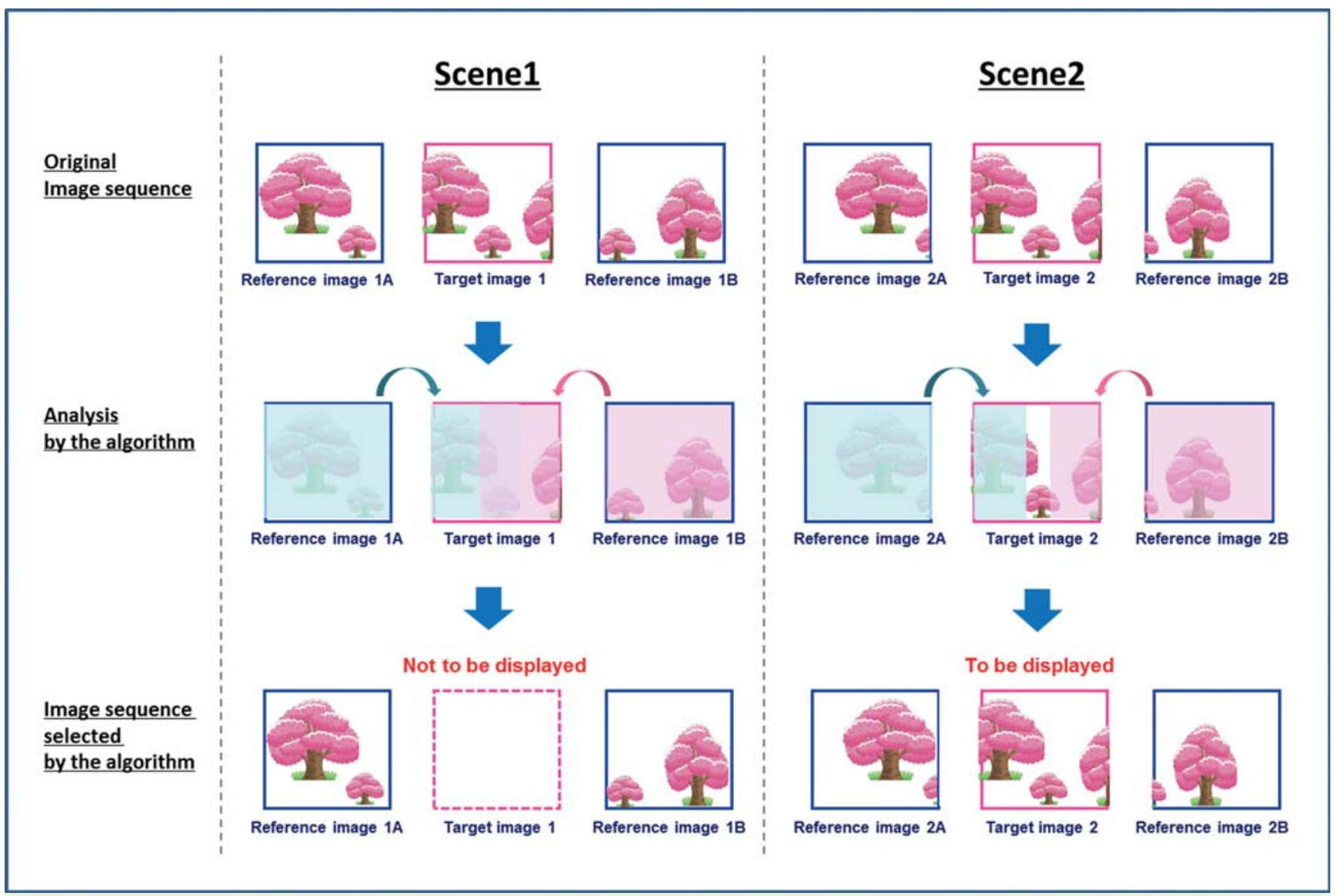

Fig. 2 Scheme of the new Omni mode algorithm. Data courtesy of Olympus Corp. The new algorithm compares the target image with reference image A and analyzes the areas of the target image covered by reference image $A$. In the same manner, it analyzes the areas of the target image covered by reference image B. If all the areas of the target image can be covered by reference images $A$ and $B$, the target image will not be displayed because it is judged as a redundant image (Scene 1). However, if there are some areas not covered by reference images $A$ and $B$ in the target image after the same comparison, the image is displayed (Scene 2).

\section{Omni mode}

The conceptual scheme for the new Omni mode algorithm is presented in $\bullet$ Fig. 2. Omni mode can select and discard redundant images and display all areas captured by VCE.

\section{Sample size calculation and statistical analysis}

The sample size was calculated such that it had an $80 \%$ power in testing hypotheses on the co-primary endpoint. With regards to the non-inferiority of the Omni mode to the control in the number of TPs in all major and minor lesions, a sample size of 40 patients was required to achieve an $80 \%$ power (alpha $=0.05$ ), assuming that the mean number of major and minor lesions following Poisson distribution was 8 (standard deviation $=2.83$ ) and the correlation coefficient between TPs within patients was 0.8 . The non-inferiority margin for the TP ratio of the Omni mode compared with the control was predefined as 0.9 . The statistical power for showing the superiority of the Omni mode over the control in reading time required was considered to be close to 1 for this sample size.

The number of TPs identified using the 2 modalities was compared using the generalized estimating equations (GEE) model with Poisson response and a log link function. The covariance structure within patients in the GEE model was compound symmetry. The fixed effects in the model were modality, time, and reader. Response variables included major and minor lesions and their composites. The reading time between modalities was compared using a general linear mixed-effects model with modality, time, and reader as the fixed effects. The covariance structure within patients in the model was compound symmetry. The Satterthwaite method was used for the adjustment of denominator degrees of freedom in a test of the fixed effects.

All statistical analyses were performed using SAS software (Version 9.2, SAS institute Inc., Cary, NC). A P value $<0.05$ was considered statistically significant.

\section{Results}

$\nabla$

The study adopted an intention-to-treat principle and 40 patients were included in the analysis. The mean age of the patients was $68.7 \pm 14.2$ years and 19 patients $(47.5 \%)$ were male. The reasons for performing VCE in each case are presented in $\bullet$ Table 1. The primary basis for performing VCE was obscure gastrointestinal bleeding. The types of predefined major and minor lesions are presented in Table 2 . A total of 264 lesions were identified in all patients, and the number of major and minor lesions identified was 71 and 193, respectively. A summary of the TPs and false positives (FPs) is presented in Table 3. Of the 264 lesions, an average of 140 and 160.5 were detected using the Omni mode and control, respectively. The estimated TP ratios and 95\% confidence intervals for total, major, and minor lesions were 0.87 (0.80-0.95), $0.93(0.83-1.04)$, and $0.83(0.74-0.94)$, respective- 
Table 1 Reasons for performing video capsule endoscopy.

\begin{tabular}{|l|l|}
\hline Reason & Number of patients \\
\hline OGIB & 29 \\
\hline Crohn's disease & 3 \\
\hline Indeterminate enteritis & 3 \\
\hline Others & 5 \\
\hline Total & 40 \\
\hline
\end{tabular}

OGIB, obscure gastrointestinal bleeding.

Table 2 Types of predefined findings of major and minor lesions on video capsule endoscopy.

\begin{tabular}{|c|c|c|}
\hline Predefined lesion & Major (n) & Minor (n) \\
\hline Ulcer & 33 & \\
\hline Angioectasia & 17 & 27 \\
\hline Bleeding & 12 & 3 \\
\hline Ulcer scar & 3 & \\
\hline Ulcer with stenosis & 2 & \\
\hline SMT & 1 & 1 \\
\hline Hemangioma & 1 & 3 \\
\hline Erosion & 1 & 47 \\
\hline Diverticulum & 1 & 2 \\
\hline Lymphangiectasia/lymphangioma & & 87 \\
\hline Tattooing & & 11 \\
\hline Red spot & & 7 \\
\hline Edema & & 1 \\
\hline Lymphoid hyperplasia & & 1 \\
\hline Hemoclip & & 1 \\
\hline Foreign body & & 1 \\
\hline Polyp & & 1 \\
\hline Total & 71 & 193 \\
\hline
\end{tabular}

SMT, submucosal tumor.

Table 3 Summary of findings of true positives and false positives.

\begin{tabular}{|c|c|c|}
\hline Endpoint & Omni mode & Control \\
\hline $\begin{array}{l}\text { Proportion of major lesions } \\
\text { detected, average } n / n \text { (ratio) }\end{array}$ & $\begin{array}{l}59 / 71 \\
(0.83)\end{array}$ & $\begin{array}{l}63.5 / 71 \\
(0.89)\end{array}$ \\
\hline $\begin{array}{l}\text { Proportion of minor lesions } \\
\text { detected, average } n / n \text { (ratio) }\end{array}$ & $\begin{array}{l}81 / 193 \\
(0.42)\end{array}$ & $\begin{array}{l}97 / 193 \\
(0.50)\end{array}$ \\
\hline $\begin{array}{l}\text { Proportion of all lesions } \\
\text { detected, average } n / n \text { (ratio) }\end{array}$ & $\begin{array}{l}140 / 264 \\
(0.53)\end{array}$ & $\begin{array}{l}160.5 / 264 \\
(0.61)\end{array}$ \\
\hline $\begin{array}{l}\text { Sensitivity for major lesion detection } \\
\text { - per patient basis, } n / n \text { (ratio) }\end{array}$ & $\begin{array}{l}51 / 56 \\
(0.90)\end{array}$ & $\begin{array}{l}53 / 56 \\
(0.95)\end{array}$ \\
\hline $\begin{array}{l}\text { Average number of false positives } \\
\text { per patient, } n\end{array}$ & 3.2 & 4.8 \\
\hline
\end{tabular}

ly. Thus, non-inferiority of the Omni mode to the control was not demonstrated in this study. In the post hoc analysis, although noninferiority was not demonstrated, the detection rate of major lesions was not significantly different when comparing the modalities. Detailed results are presented in $\bullet$ Table 4. The mean numbers of FPs were 127.5 (3.19/patient) and 192.5 (4.81/patient) using the Omni mode and control, respectively. Although the readers detected a high number of true lesions using the control, they also detected a high number of FPs. We calculated the proportion of readings with the detection of 1 or more major lesions from the total cases in which they were present and found that the proportions were $90 \%$ using the Omni mode and $95 \%$ using the control. All undetected minor lesions were subtle lesions that did not influence the therapeutic decisions.
Table 4 Primary outcome in the GEE Poisson models ${ }^{1}$.

\begin{tabular}{|ll}
\hline Variable & Ratio estimate (CI) \\
\hline Detected major lesions & $0.93(0.83-1.04)$ \\
\hline Detected minor lesions & $0.83(0.74-0.94)$ \\
\hline Total detected lesions & $0.87(0.80-0.95)$ \\
\hline
\end{tabular}

$\mathrm{Cl}$, confidence interval.

${ }^{1}$ Outcome modeled using Poisson distribution with identity link. The ratio estimate is Omni mode compared with control.

Table 5 Secondary outcome, general linear mixed model.

\begin{tabular}{|ll}
\hline Variable & Estimate $(\mathrm{Cl})^{1}$ \\
\hline Reading time (minutes) & $-47.7(-52.0,-43.4)$
\end{tabular}

$\mathrm{Cl}$, confidence interval.

${ }^{1}$ Estimate reported is a difference between the Omni mode with control as a reference.

Regarding the reading time, this was significantly different across the modalities $(P<0.001)$, with the Omni mode requiring 27.3 minutes and the control requiring 75.1 minutes, on average. The difference in reading time between modalities was calculated as approximately 47.7 minutes ( $\bullet$ Table 5 ).

The total number of displayed images per video of the small intestine was $13,444 \pm 4341$ using the Omni mode and 39,377 \pm 13,568 using the control. Therefore, a $65 \%$ reduction in the number of displayed images was achieved using the Omni mode. In addition, after calculating the software-associated false negatives for major lesions, we noted that the Omni mode was able to identify all predefined major lesions, and no software-associated false negatives were recorded for this modality.

\section{Discussion}

The non-inferiority of the Omni mode to a control was not demonstrated in the current study. However, the reading time was found to be significantly lower on using the Omni mode than on using the control.

In order to reduce the possibility of missing lesions and the reading time required, several software modes have been developed including the Blood indicator [10-12], Blue mode [13,14], Flexible spectral Imaging Color Enhancement (FICE) [15], Automatic mode [7], and Quickview $[8,16]$. The Blood indicator selects red (blood) images [10-12] while Blue mode enhances lesions that are blue $[14,15]$. FICE is based on the arithmetic narrowing of the bandwidth of a conventional endoscopic image using computerized spectral estimation technology to enhance the lesion, thus simplifying detection [17]. In Automatic mode, the software reduces the total number of images by combining similar images [7]; while in Quickview, the software reduces the number of recorded images by identifying the most unusual images and presenting selected images $[8,16]$. The Omni mode is a new algorithm that selects and discards redundant images and displays all areas captured by VCE. Several previous single-center pilot studies have demonstrated the usefulness of these modes on a limited number of patients. The current study included 7 expert VCE readers, 3 judging committee members, an expert biostatistician, and a large sample size of patients. First, we attempted to confirm the non-inferiority of the Omni mode against a control for detecting lesions in a calculated and specified sample. On analysis, we were unable to demonstrate the non-inferiority of the Omni 
mode; however, we found that the detection rate of major lesions was not significantly different with the Omni mode when compared with the control. We suspect that our failure in confirming the Omni mode's non-inferiority resulted from an under-powered sample size and heterogeneity in the definition of lesions. While we had entered the expected number of major and minor lesions into the power analysis, the number of lesions in each video was too small to determine non-inferiority. Moreover, among the minor lesions, heterogeneity in the definition of lesions was recognized. For example, one reader detected a small lymphangiectasia as a minor lesion, while another reader considered the same lesion a normal finding. Low rates of TPs and high rates of FPs were observed among the minor lesions. In a future study, we intend to calculate the sample size using the number of major lesions, as these are considered abnormal.

The reading time was significantly lower when using the Omni mode than when using the control. In the post hoc analysis, the rate of TPs among major lesions was not significantly different between modalities. While these findings could not directly confirm equivalence between the Omni mode and control, the findings suggest that the Omni mode may reduce the reading time without decreasing the rate of TPs. A further clinical trial that aims to assess more than 8 definitive major lesions is required to support these findings.

The Omni mode could reduce the redundancy and sequentiality of the VCE video. However, the readers in the current study did not have experience using the Omni mode. We evaluated the types of false negatives identified in the Omni mode and the control and found that types of missed lesions did not differ across the modalities. However, variations in the number of false negatives identified with each modality appeared to be derived from the reader's abilities. Thus, reader training is required for the operation of Omni mode and reading parameters, such as video frame rate and display of dual or quad images, must be optimized.

A potential advantage of the Omni mode is its capacity to act as a filter and identify patients who need a detailed, standard video reading. In other words, if the Omni mode is "positive" it may be unnecessary to devote an extended period of time to the video review using the standard video reading mode. On the other hand, considering the performance of the Omni mode, it is possible that a "negative" capsule in Omni mode could be false. Thus, if a capsule is identified as positive using Omni mode, there is no need to review the entire video using standard mode. Interestingly, the Omni mode was able to identify all predefined major lesions and software-associated false negatives were not recognized. Therefore, the Omni mode could reduce VCE reading time without increasing the lesion miss rate.

In conclusion, although the non-inferiority of the Omni mode to a control was not demonstrated, the detection rate for major lesions was not significantly different between the modalities. Furthermore, required reading time was low with the Omni mode. Therefore, Omni mode may be appropriate only for assessment of major lesions using VCE after optimization of the reading parameters and thorough reader training on the use of this modality.

Competing interest: The capsule endoscopy software and workstation were provided by Olympus. Dr. Ogata received a research grant from Olympus.

\section{Institutions}

${ }^{1}$ Center for Diagnostic and Therapeutic Endoscopy, School of Medicine, Keio University, Tokyo, Japan

2 Department of Gastroenterology, Osaka City University Graduate School of
Medicine, Osaka, Japan

3 Third Department of Internal Medicine, Kansai Medical University, Osaka, Japan

${ }^{4}$ Department of Medicine and Clinical Science, Graduate School of Medical Sciences, Kyushu University, Fukuoka, Japan

${ }^{5}$ Division of Gastroenterology, Department of Internal Medicine, School of Medicine, Iwate Medical University, Morioka, Japan

${ }^{6}$ Department of Preventive Medicine and Public Health, Biostatistics at Center for Clinical Research, Keio University School of Medicine, Tokyo, Japan

Division of Gastroenterology and Hepatology, Department of Internal

Medicine, Keio University School of Medicine, Tokyo, Japan

${ }^{8}$ Department of Gastroenterology, School of Medicine, Tokyo Medical and Dental University, Tokyo, Japan

${ }^{9}$ Department of Endoscopy, The jikei University School of Medicine, Tokyo, Japan

10 Department of Gastroenterology, School of Medicine, Fujita Health University, Aichi, Japan

${ }^{11}$ Department of Gastroenterology, Nagoya Graduate School of Medicine, Nagoya, Japan

${ }^{12}$ Comprehensive Internal Medicine, Shiga University of Medical Science, Shiga, Japan

\section{References}

1 Triester SL, Leighton JA, Leontiadis GI et al. A meta-analysis of the yield of capsule endoscopy compared to other diagnostic modalities in patients with obscure gastrointestinal bleeding. Am J Gastroenterol 2005; 100: $2407-2418$

2 Min YW, Kim JS, Jeon SW et al. Long-term outcome of capsule endoscopy in obscure gastrointestinal bleeding: a nationwide analysis. Endoscopy 2014; 46: 59-65

3 Papadakis KA, Lo SK, Fireman Z et al. Wireless capsule endoscopy in the evaluation of patients with suspected or known Crohn's disease. Endoscopy 2005; 37: 1018-1022

4 Waterman M, Gralnek IM. Capsule endoscopy of the esophagus. J Clin Gastroenterol 2009; 43: 605-612

5 Rey JF, Ogata $H$, Hosoe $N$ et al. Blinded nonrandomized comparative study of gastric examination with a magnetically guided capsule endoscope and standard videoendoscope. Gastrointest Endosc 2012; 75: $373-381$

6 Spada C, Hassan C, Munoz-Navas M et al. Second-generation colon capsule endoscopy compared with colonoscopy. Gastrointest Endosc 2011; 74 : 581 - 589.e581

7 Hosoe N, Rey JF, Imaeda $\mathrm{H}$ et al. Evaluations of capsule endoscopy software in reducing the reading time and the rate of false negatives by inexperienced endoscopists. Clin Res Hepatol Gastroenterol 2012; 36: $66-71$

8 Shiotani A, Honda K, Kawakami M et al. Evaluation of RAPID((R)) 5 Access software for examination of capsule endoscopies and reading of the capsule by an endoscopy nurse. J Gastroenterol 2011; 46: 138 - 142

9 Subramanian V, Mannath J, Telakis E et al. Efficacy of new playback functions at reducing small-bowel wireless capsule endoscopy reading times. Dig Dis Sci 2012; 57: 1624-1628

10 Stein AC, Appannagari A, Habib I et al. A rapid and accurate method to detect active small bowel gastrointestinal bleeding on video capsule endoscopy. Dig Dis Sci 2014; 59: 2503-2507

11 D'Halluin PN, Delvaux M, Lapalus MG et al. Does the "Suspected Blood Indicator" improve the detection of bleeding lesions by capsule endoscopy? Gastrointest Endosc 2005; 61: 243-249

12 Liangpunsakul S, Mays L, Rex DK. Performance of Given suspected blood indicator. Am J Gastroenterol 2003; 98: 2676-2678

13 Koulaouzidis A, Smirnidis A, Douglas S et al. QuickView in small-bowel capsule endoscopy is useful in certain clinical settings, but QuickView with Blue Mode is of no additional benefit. Eur J Gastroenterol Hepatol 2012; 24: $1099-1104$

14 Krystallis C, Koulaouzidis A, Douglas S et al. Chromoendoscopy in small bowel capsule endoscopy: Blue mode or Fuji Intelligent Colour Enhancement? Dig Liver Dis 2011; 43: 953 -957

15 Nakamura $M$, Ohmiya $N$, Miyahara $R$ et al. Usefulness of flexible spectral imaging color enhancement (FICE) for the detection of angiodysplasia in the preview of capsule endoscopy. Hepato-gastroenterology 2012; 59: $1474-1477$

16 Westerhof J, Koornstra JJ, Weersma RK. Can we reduce capsule endoscopy reading times? Gastrointest Endosc 2009; 69: 497 - 502

17 Pohl J, Aschmoneit I, Schuhmann $S$ et al. Computed image modification for enhancement of small-bowel surface structures at video capsule endoscopy. Endoscopy 2010; 42: 490-492 